\title{
Effects of Beak Trimming, Stocking Density and Sex on Carcass Yield, Carcass Components, Plasma Glucose and Triglyceride Levels in Large White Turkeys
}

\author{
Turgay Sengul, Hakan Inci*, Ahmet Y. Sengul, Bunyamin Sogut, and Selahattin Kiraz ${ }^{1}$ \\ Animal Science Department, Agricultural Faculty, Bingöl University, Bingöl 12000, Turkey \\ ${ }^{1}$ Animal Science and Nutrition Department, Agricultural Faculty, Harran University, Sanliurfa 63300, Turkey
}

\begin{abstract}
This study was conducted to determine the effects of beak trimming, stocking density (D) and sex (S) on live weight (LW), carcass yield and its component, and plasma glucose (PG) and triglyceride levels in Large White turkeys. To accomplish this aims, totally 288 $\mathrm{d}$ old large white turkey chicks (144 in each sex) were used. Beaks of 77 male and female poults were trimmed when $8 \mathrm{~d}$ old with an electrical beak trimmer. The birds were fed by commercial turkey rasion. Experiment was designed as $2 \times 2 \times 2$ factorial arrangement with 3 replications in each group. Beak trimming and stocking density did not affect live weight, carcass composition and its components. The higher LW and carcass weight observed in trimmed groups. As expected, male birds are heavier than female, and carcass percentage (CP) would be adverse. However, in this study, CP of male was higher in trimmed, in $0.25 \mathrm{~m}^{2} /$ bird. (D) $\times$ sex (S) interaction had an effect on both $\mathrm{CP}$ and thigh weights $(p<0.05)$. Significantly $\mathrm{D} \times \mathrm{S}$ was observed in LW, CP and PG. The weight of carcass and its some components were higher in male. $\mathrm{S} \times \mathrm{D}$ interaction had an effect on plasma glucose level $(p<0.05)$. Triglyceride level was affected $(p<0.05)$ by sex. Significant relationships were found between percentage of thighs $(\mathrm{r}=0.447, p<0.01)$ and percentage of breast $(\mathrm{r}=0.400$, $p<0.01$ ). According to this study, it can be said that trimming is useful with density of $0.25 \mathrm{~m}^{2} /$ bird in turkey fattening.
\end{abstract}

Keywords: turkeys, beak trimming, stocking density, sex, carcass characteristics

Received May 8, 2014; Revised October 17, 2014; Accepted December 10, 2014

\section{Introduction}

In poultry, carcass yield and carcass components are affected by such factors as genotype, sex, age, nutrition, and climate (Brake et al., 1995; Leenstra, 1986; Sell et al., 1985; Veldkamp et al., 2000a). Additionally, processing in slaughter has an important effect on carcass characteristics as well.

In turkey production, beak trimming, stocking density, and sex are factors affecting carcass yield and components (Cunningham et al., 1992). The advantages of beak trimming are; stopping finger and tail pecking in chicks, preventing vent and comb pecking in adults, reducing fighting's among males, stopping eating eggs in female, reducing the stress in flock, controlling cannibalism, preventing feed waste, increasing the feed conversion and

\footnotetext{
*Corresponding author: Hakan Inci, Bingol University, Agricultural Faculty, Animal Science Department, 12100 Bingöl, Turkey. Tel. +90-4262160030, Fax: +90-4262160029, E-mail: hakaninci 2565@hotmail.com
}

the livability, and providing a higher uniformity in flock (North and Bell, 1990). Debeaking is being done as a routine procedure in turkeys industry (Cunningham et al., 1992). It has been reported that turkeys' beaks should be trimmed if housed in a confinement area. It is also advised that beak trimming should be done during the first three weeks in poultry (Ensminger, 1992). Welfare and comfort supplied with optimum stocking density $(2.5$ turkeys $/ \mathrm{m}^{2}$ ) affect their health and performance positively. The bird numbers that are higher or less than optimum number in a per unit area decreases production, thus the number of birds affects the farms' profits negatively. Stocking density was significantly affected by season, housing isolation, and ventilation factors (Parkhurts and Mountney, 1988). In general, it is suggested that the stocking density for turkeys should be $0.4 \mathrm{~m}^{2}$ per bird (Ensminger, 1992).

It is well known that carcass weight and its components of turkeys were affected by sex more than other poultry species. Because male turkeys grow faster than females; they reach heavier live weight and carcass weight at the

@This is an open access article distributed under the terms of the Creative Commons Attribution Non-Commercial License (http://creativecommons.org/licences/ by-nc/3.0) which permits unrestricted non-commercial use, distribution, and reproduction in any medium, provided the original work is properly cited. 
same duration. Fattening of carcass in turkeys is started earlier by females.

In this study, the effects of beak trimming, stocking density and sex on carcass yield and components, edible yields, abdominal fat, plasma glucose, and triglyceride levels in large white turkeys and the relationships among these characteristics were investigated.

\section{Materials and Methods}

Total $288 \mathrm{~d}$ old large white turkey chicks (144 in each sex) were used in this study. Poults' sex separation were done at the first day and were kept in brooding machines during the first two weeks. Beaks of seventy-seven male and female poults were trimmed when $8 \mathrm{~d}$ old with an electrical beak trimmer. Experiment was designed as $2 \times$ $2 \times 2$ factorial arrangement with 3 replications in each group. Poults were placed into 24 pens at the end of the second week. Stocking density was 0.25 and $0.50 \mathrm{~m}^{2}$ per bird; as the stocking densities in each pan and had $3.0 \mathrm{~m}^{2}$ $(1.5 \times 2 \mathrm{~m})$ and $6.0 \mathrm{~m}^{2}(3 \times 2 \mathrm{~m})$ spaces in which 12 birds were placed. Wood shaving was used as litter. Feed and water requirements of birds were supplied by ad libitum. Birds were fed with diets containing $28 \%$ crude protein and $2800 \mathrm{kcal} / \mathrm{kg} \mathrm{ME}$ at $0-4$ wk periods, $26 \%$ crude protein and $2900 \mathrm{kcal} / \mathrm{kg}$ ME at $5-8$ wk periods, $22 \%$ crude protein and $3000 \mathrm{kcal} / \mathrm{kg} \mathrm{ME}$ at $9-14$ wk periods, and $19 \%$ crude protein and $3100 \mathrm{kcal} / \mathrm{kg}$ ME at $15-18 \mathrm{wk}$ periods. Lighting during the first two weeks was provided daily as $23 \mathrm{~L}: 1 \mathrm{D}$ and then $16 \mathrm{~L}: 8 \mathrm{D}$ till the end of the experiment.

Totally 48 birds (two birds from each pen) were slaughtered at the end of 18th wk. Then, turkeys were fasted for $12 \mathrm{~h}$. Their carcass, thigh, breast, ridge, wing, neck, heart, liver, gizzard, and abdominal fat weights were measured with a digital scale $( \pm 1 \mathrm{~g}$ sensitivity). Blood samples collected from birds during slaughtering were analyzed in a biochemistry laboratory. Plasma triglyceride and glucose levels were determined by auto analyzer (Olimpus AU 2700).

Statistical analysis was carried out by using SPSS 10.0 software program and for the comparison of the mean values; the Duncan tests range was utilized.

\section{Results}

Beak trimming and stocking density had no significant effect on live weights, carcass weights, carcass percentage, plasma triglyceride and glucose levels, the carcass components (breast, thigh, ridge, wing and neck) and their percentages in whole carcass, liver, gizzard and abdominal fat weights. However, Some characteristics such as carcass percentage, ridge, breast, liver and gizzard; live weight, carcass weight, parts of carcass percentage, edible yields and abdominal were significantly affected $(p<0.05)$ by sex (Table 1 and 2 ).

Table 1. Effect of beak trimming, stocking density and sex on live weight, carcass weight, carcass percentage, plasma triglyceride and glucose levels

\begin{tabular}{|c|c|c|c|c|c|c|c|}
\hline Beak trimming & $\begin{array}{l}\text { Density } \\
\left(\mathrm{m}^{2} / \text { bird }\right)\end{array}$ & Sex & $\begin{array}{c}\text { Live weight } \\
(\mathrm{kg})\end{array}$ & $\begin{array}{c}\text { Carcass weight } \\
(\mathrm{kg})\end{array}$ & $\begin{array}{c}\text { Carcass percent- } \\
\text { age }(\%)\end{array}$ & $\begin{array}{l}\text { Triglyceride } \\
(\mathrm{mg} / \mathrm{dL})\end{array}$ & $\begin{array}{l}\text { Glucose } \\
(\mathrm{mg} / \mathrm{dL})\end{array}$ \\
\hline \multirow{4}{*}{ Untrimmed } & \multirow{2}{*}{0.25} & $\mathrm{M}$ & $8.56^{\mathrm{a}}$ & $6.25^{\mathrm{a}}$ & $72.8^{\mathrm{a}}$ & $70.8^{\mathrm{a}}$ & $265^{\mathrm{a}}$ \\
\hline & & $\mathrm{F}$ & $8.37^{\mathrm{a}}$ & $6.29^{\mathrm{a}}$ & $75.1^{\mathrm{ab}}$ & $81.3^{\mathrm{a}}$ & $284^{\mathrm{ab}}$ \\
\hline & \multirow{2}{*}{0.50} & M & $9.24^{\mathrm{abc}}$ & $6.99^{\mathrm{ab}}$ & $75.7^{\mathrm{ab}}$ & $76.8^{\mathrm{a}}$ & $269^{\mathrm{a}}$ \\
\hline & & $\mathrm{F}$ & $8.79^{\mathrm{ab}}$ & $6.68^{\mathrm{a}}$ & $76.0^{\mathrm{ab}}$ & $86.3^{\mathrm{ab}}$ & $278^{\mathrm{ab}}$ \\
\hline \multirow{4}{*}{ Trimmed } & \multirow{2}{*}{0.25} & M & $9.87^{\mathrm{c}}$ & $7.61^{\mathrm{b}}$ & $77.0^{\mathrm{b}}$ & $70.2^{\mathrm{a}}$ & $298^{\mathrm{b}}$ \\
\hline & & $\mathrm{F}$ & $8.35^{\mathrm{a}}$ & $6.26^{\mathrm{a}}$ & $75.0^{\mathrm{ab}}$ & $75.2^{\mathrm{a}}$ & $279^{\mathrm{ab}}$ \\
\hline & \multirow{2}{*}{0.50} & M & $9.62^{\mathrm{bc}}$ & $7.14^{\mathrm{ab}}$ & $73.8^{\mathrm{ab}}$ & $74.2^{\mathrm{a}}$ & $277^{\mathrm{ab}}$ \\
\hline & & $\mathrm{F}$ & $8.36^{\mathrm{a}}$ & $6.34^{\mathrm{a}}$ & $75.9^{\mathrm{ab}}$ & $110.8^{\mathrm{b}}$ & $272^{\mathrm{ab}}$ \\
\hline \multicolumn{2}{|c|}{ SEM } & & 0.32 & 0.30 & 1.14 & 9.5 & 8.8 \\
\hline \multicolumn{8}{|c|}{ Source of variation } \\
\hline \multicolumn{3}{|c|}{ Beak trimming (B) } & NS & NS & NS & NS & NS \\
\hline \multicolumn{3}{|c|}{ Stoking density (D) } & NS & NS & NS & NS & NS \\
\hline \multicolumn{3}{|c|}{$\operatorname{Sex}(S)$} & $* * *$ & $* *$ & NS & $*$ & NS \\
\hline \multicolumn{3}{|c|}{$\mathrm{B} \times \mathrm{D}$} & NS & NS & NS & NS & NS \\
\hline \multicolumn{3}{|c|}{$\mathrm{B} \times \mathrm{S}$} & NS & NS & NS & NS & NS \\
\hline \multicolumn{3}{|c|}{$\mathrm{D} \times \mathrm{S}$} & $*$ & NS & * & NS & $*$ \\
\hline \multicolumn{3}{|c|}{$\mathrm{B} \times \mathrm{D} \times \mathrm{S}$} & NS & NS & NS & NS & NS \\
\hline
\end{tabular}

${ }^{\mathrm{a}-\mathrm{c}}$ Means in column with different superscripts differ significantly. ${ }^{*} p<0.05, * * p<0.01,{ }^{*} * * p<0.001$

M, male; F, female; NS, Non significant; SEM, Standart error of means. 
Table 2. Effect of beak trimming, stocking density and sex on carcass components

\begin{tabular}{|c|c|c|c|c|c|c|c|c|c|c|c|c|}
\hline \multicolumn{3}{|c|}{ Treatments } & \multicolumn{5}{|c|}{ \% of carcass } & \multicolumn{5}{|c|}{$\overline{\text { Weight (kg) }}$} \\
\hline Beak trimming & $\begin{array}{l}\text { Density } \\
\left(\mathrm{m}^{2} / \text { bird }\right)\end{array}$ & Sex & Breast & Thighs & Wings & Ridge & Neck & Breast & Thighs & Wings & Ridge & Neck \\
\hline \multirow{4}{*}{ Untrimmed } & \multirow{2}{*}{0.25} & $\mathrm{M}$ & $29.7^{\mathrm{a}}$ & $42.4^{\mathrm{b}}$ & $13.4^{\mathrm{b}}$ & $7.8^{\mathrm{ab}}$ & $5.2^{\mathrm{bc}}$ & 1.86 & $2.65^{\mathrm{a}}$ & $0.84^{\text {bc }}$ & $0.49^{\mathrm{a}}$ & $0.32^{\mathrm{ab}}$ \\
\hline & & $\mathrm{F}$ & $33.2^{\mathrm{b}}$ & $40.1^{\mathrm{a}}$ & $11.8^{\mathrm{a}}$ & $7.9^{\mathrm{ab}}$ & $5.0^{\mathrm{abc}}$ & 2.09 & $2.52^{\mathrm{a}}$ & $0.74^{\mathrm{ab}}$ & $0.50^{\mathrm{b}}$ & $0.31^{\mathrm{a}}$ \\
\hline & \multirow{2}{*}{0.50} & $\mathrm{M}$ & $30.4^{\mathrm{a}}$ & $40.7^{\mathrm{ab}}$ & $13.6^{\mathrm{b}}$ & $8.2^{\mathrm{ab}}$ & $5.2^{\mathrm{bc}}$ & 2.13 & $2.84^{\mathrm{abc}}$ & $0.95^{\mathrm{d}}$ & $0.57^{\mathrm{ab}}$ & $0.36^{\mathrm{bc}}$ \\
\hline & & $\mathrm{F}$ & $33.3^{\mathrm{b}}$ & $40.6^{\mathrm{ab}}$ & $11.6^{\mathrm{a}}$ & $7.7^{\mathrm{a}}$ & $4.7^{\mathrm{ab}}$ & 2.23 & $2.71^{\mathrm{ab}}$ & $0.78^{\mathrm{ab}}$ & $0.51^{\mathrm{a}}$ & $0.31^{\mathrm{a}}$ \\
\hline \multirow{4}{*}{ Trimmed } & \multirow{2}{*}{0.25} & $\mathrm{M}$ & $31.3^{\mathrm{a}}$ & $40.7^{\mathrm{ab}}$ & $13.0^{\mathrm{b}}$ & $8.4^{\mathrm{b}}$ & $4.9^{\mathrm{abc}}$ & 2.39 & $3.09^{c}$ & $0.99^{\mathrm{d}}$ & $0.64^{\mathrm{b}}$ & $0.37^{\mathrm{c}}$ \\
\hline & & $\mathrm{F}$ & $33.6^{\mathrm{b}}$ & $40.0^{\mathrm{a}}$ & $11.6^{\mathrm{a}}$ & $8.3^{\mathrm{ab}}$ & $4.5^{\mathrm{a}}$ & 2.11 & $2.50^{\mathrm{a}}$ & $0.73^{\mathrm{a}}$ & $0.52^{\mathrm{a}}$ & $0.28^{\mathrm{a}}$ \\
\hline & \multirow{2}{*}{0.50} & $\mathrm{M}$ & $29.9^{\mathrm{a}}$ & $42.0^{\mathrm{b}}$ & $13.3^{\mathrm{b}}$ & $7.7^{\mathrm{ab}}$ & $5.4^{\mathrm{c}}$ & 2.15 & $2.99^{\mathrm{bc}}$ & $0.94^{\mathrm{cd}}$ & $0.56^{\mathrm{ab}}$ & $0.37^{\mathrm{c}}$ \\
\hline & & $\mathrm{F}$ & $33.8^{\mathrm{b}}$ & $39.8^{\mathrm{a}}$ & $11.7^{\mathrm{a}}$ & $7.8^{\mathrm{ab}}$ & $4.9^{\mathrm{abc}}$ & 2.15 & $2.53^{\mathrm{a}}$ & $0.74^{\mathrm{ab}}$ & $0.49^{\mathrm{a}}$ & $0.31^{\mathrm{a}}$ \\
\hline \multicolumn{2}{|c|}{ SEM } & & 0.6 & 0.6 & 0.2 & 0.2 & 0.2 & 0.12 & 0.11 & 0.04 & 0.03 & 0.02 \\
\hline \multicolumn{13}{|c|}{ Source of variation } \\
\hline \multicolumn{3}{|c|}{ Beak trimming (B) } & NS & NS & NS & NS & NS & NS & NS & NS & NS & NS \\
\hline \multicolumn{3}{|c|}{ Stocking density (D) } & NS & NS & NS & NS & NS & NS & NS & NS & NS & NS \\
\hline \multicolumn{3}{|c|}{$\operatorname{Sex}(S)$} & $* * *$ & $* *$ & $* * *$ & NS & $* *$ & NS & $* * *$ & $* * *$ & $* *$ & $* * *$ \\
\hline \multicolumn{3}{|c|}{$\mathrm{B} \times \mathrm{D}$} & NS & NS & NS & $*$ & $*$ & NS & NS & NS & $*$ & NS \\
\hline \multicolumn{3}{|c|}{$\mathrm{B} \times \mathrm{S}$} & NS & NS & NS & NS & NS & NS & NS & NS & NS & NS \\
\hline \multicolumn{3}{|c|}{$\mathrm{D} \times \mathrm{S}$} & NS & NS & NS & NS & NS & NS & $*$ & NS & NS & $*$ \\
\hline \multicolumn{3}{|c|}{$\mathrm{B} \times \mathrm{D} \times \mathrm{S}$} & NS & * & NS & NS & NS & NS & NS & NS & NS & NS \\
\hline
\end{tabular}

${ }^{\mathrm{a}-\mathrm{c}}$ Means in column with different superscripts differ significantly. ${ }^{*} p<0.05,{ }^{* *} p<0.01,{ }^{* * *} p<0.001$

$\mathrm{M}$, male; F, female; NS, Non significant; SEM, Standart error of means.

Table 3. Effect of beak trimming, stocking density and sex on edible yields and abdominal fat

\begin{tabular}{|c|c|c|c|c|c|c|c|c|c|c|}
\hline \multicolumn{3}{|c|}{ Treatments } & \multicolumn{4}{|c|}{ \% of carcass } & \multicolumn{4}{|c|}{ Weights (g) } \\
\hline Beak trimming & $\begin{array}{l}\text { Density } \\
\left(\mathrm{m}^{2} / \text { bird }\right)\end{array}$ & Sex & Liver & Gizzard & Heart & $\begin{array}{l}\text { Abdominal } \\
\text { fat }\end{array}$ & Liver & Gizzard & Heart & $\begin{array}{l}\text { Abdominal } \\
\text { fat }\end{array}$ \\
\hline \multirow{4}{*}{ Untrimmed } & \multirow{2}{*}{0.25} & $\mathrm{M}$ & $1.73^{b}$ & $2.53^{b}$ & $0.47^{b}$ & $0.11^{\mathrm{a}}$ & $107^{\mathrm{bc}}$ & 156 & $29^{\mathrm{a}}$ & $7.7^{\mathrm{a}}$ \\
\hline & & $\mathrm{F}$ & $1.67^{\mathrm{b}}$ & $2.62^{\mathrm{b}}$ & $0.40^{\mathrm{ab}}$ & $0.82^{\mathrm{bc}}$ & $105^{\mathrm{abc}}$ & 165 & $26^{\mathrm{a}}$ & $51.2^{\mathrm{bc}}$ \\
\hline & \multirow{2}{*}{0.50} & M & $1.62^{\mathrm{ab}}$ & $2.22^{\mathrm{ab}}$ & $0.42^{\mathrm{ab}}$ & $0.23^{\mathrm{a}}$ & $112^{\mathrm{bc}}$ & 154 & $30^{\mathrm{a}}$ & $16.3^{\mathrm{a}}$ \\
\hline & & $\mathrm{F}$ & $1.37^{\mathrm{a}}$ & $2.25^{\mathrm{ab}}$ & $0.38^{\mathrm{a}}$ & $0.91^{\mathrm{c}}$ & $90^{\mathrm{a}}$ & 149 & $25^{\mathrm{a}}$ & $61.3^{\mathrm{c}}$ \\
\hline \multirow{4}{*}{ Trimmed } & \multirow{2}{*}{0.25} & M & $1.58^{\mathrm{ab}}$ & $2.07^{\mathrm{a}}$ & $0.47^{\mathrm{b}}$ & $0.15^{\mathrm{a}}$ & $120^{\mathrm{c}}$ & 156 & $35^{\mathrm{b}}$ & $12.0^{\mathrm{a}}$ \\
\hline & & $\mathrm{F}$ & $1.67^{\mathrm{b}}$ & $2.48^{\mathrm{ab}}$ & $0.40^{\mathrm{ab}}$ & $0.67^{\mathrm{bc}}$ & $104^{\mathrm{ab}}$ & 156 & $24^{\mathrm{a}}$ & $41.8^{\mathrm{b}}$ \\
\hline & \multirow{2}{*}{0.50} & M & $1.50^{\mathrm{ab}}$ & $2.08^{\mathrm{a}}$ & $0.35^{\mathrm{a}}$ & $0.18^{\mathrm{a}}$ & $106^{\mathrm{abc}}$ & 145 & $25^{\mathrm{a}}$ & $14.0^{\mathrm{a}}$ \\
\hline & & $\mathrm{F}$ & $1.63^{\mathrm{b}}$ & $2.45^{\mathrm{ab}}$ & $0.38^{\mathrm{a}}$ & $0.60^{\mathrm{b}}$ & $102^{\mathrm{ab}}$ & 154 & $24^{\mathrm{a}}$ & $39.2^{\mathrm{b}}$ \\
\hline \multicolumn{3}{|c|}{ SEM } & 0.08 & 0.14 & 0.02 & 0.09 & 5.1 & 6.6 & 1.6 & 6.4 \\
\hline \multicolumn{11}{|c|}{ Source of variation } \\
\hline \multicolumn{3}{|c|}{ Beak trimming (B) } & $*$ & NS & $*$ & NS & NS & NS & $*$ & NS \\
\hline \multicolumn{3}{|c|}{ Stoking density (D) } & NS & NS & NS & NS & NS & NS & NS & NS \\
\hline \multicolumn{3}{|c|}{$\operatorname{Sex}(S)$} & NS & $*$ & $*$ & $* * *$ & $* *$ & NS & $* * *$ & $* * *$ \\
\hline \multicolumn{3}{|c|}{$\mathrm{B} \times \mathrm{D}$} & NS & NS & NS & NS & NS & NS & $*$ & NS \\
\hline \multicolumn{3}{|c|}{$\mathrm{B} \times \mathrm{S}$} & NS & NS & $*$ & NS & NS & NS & NS & NS \\
\hline \multicolumn{3}{|c|}{$\mathrm{D} \times \mathrm{S}$} & $*$ & NS & NS & NS & NS & NS & NS & NS \\
\hline \multicolumn{3}{|c|}{$\mathrm{B} \times \mathrm{D} \times \mathrm{S}$} & NS & NS & NS & NS & $*$ & NS & $* *$ & NS \\
\hline
\end{tabular}

${ }^{\mathrm{a}-\mathrm{c}}$ Means in column with different superscripts differ significantly. ${ }^{*} p<0.05,{ }^{* *} p<0.01,{ }^{* * *} p<0.001$

M, male; F, female; NS, Non significant; SEM, Standart error of means.

Beak trimming $\times$ stocking density interaction affected significantly $(p<0.05)$ percentage of ridge, ridge weight, percentage of neck and heart weight. Percentage of heart in whole carcass were affected significantly $(p<0.05)$ by the beak trimming $\times$ sex interaction (Table 3 ). The effects on live weight, carcass percentage, plasma glucose level, thigh weights, neck weight, and percentage of liver in whole carcass of stocking density $\times$ sex interaction were found to be significant $(p<0.05)$. Similarly, beak trimming $\times$ stocking density $\times$ sex interaction had a significant $(p<0.01)$ effect on the percentages of thighs in whole carcass, liver weight $(p<0.05)$ and heart weight. 
The correlations between carcass percentage with thigh percentage $(\mathrm{r}=-0.447, p<0.01)$, breast percentage $(\mathrm{r}=-0.400$, $p<0.01)$ and ridge percentage $(\mathrm{r}=0.363, p<0.05)$ were found to be significant (Table 4). Similarly, the correlations between thigh weights with live weight, carcass weight and breast weight were found to be significant $(p<0.001)$. Positive and significant correlations were determined between plasma triglyceride level with abdominal fat weight $(\mathrm{r}=0.315)$ and abdominal fat percentage $(\mathrm{r}=0.309)$; between plasma glucose level with carcass weight $(\mathrm{r}=0.285)$, breast weight $(\mathrm{r}=0.301)$ and neck weight (Table 5). But, negative and significant correlations were determined between plasma glucose levels with percentage of wings.

\section{Discussion}

In this study, sex (S) had the predominant effect on live weight, carcass components, edible yields, and abdominal fat with plasma triglyceride and glucose levels in turkeys. Brake et al. (1995) and Lilburn and Nestor (1991) stated that there were significant differences between sexes and lines in respect to carcass weight, carcass components and abdominal fat. In this study, carcass percentage and abdominal fat weight for male and female were found lower for males than females. Finding the highest carcass percentage for male in $0.25 \mathrm{~m}^{2} /$ bird in beak trimming (B), however, indicates that trimming had an advantage for carcass percentage even though it was statistically not important. Observing the heaviest live weight in trimmed

Table 4. Correlations between carcass percentages, percentages of carcass components, percentages of edible yields, percentages of abdominal fat, plasma triglyceride and glucose levels

\begin{tabular}{|c|c|c|c|c|c|c|c|c|c|c|c|}
\hline & Breast & Thighs & Wings & Ridge & Neck & Liver & Gizzard & Heart & $\begin{array}{l}\text { Abdomi- } \\
\text { nal fat }\end{array}$ & $\begin{array}{l}\text { Trigly- } \\
\text { ceride } \\
(\mathrm{mg} / \mathrm{dL})\end{array}$ & $\begin{array}{l}\text { Glucose } \\
\text { (mg/dL) }\end{array}$ \\
\hline $\begin{array}{c}\text { Carcass } \\
\text { percentage }\end{array}$ & $0.400 * *$ & $-0.447 * *$ & -0.214 & $0.363^{*}$ & $-0.386^{* *}$ & $-0.416^{* *}$ & $-0.495 * * *$ & -0.129 & 0.252 & 0.145 & 0.231 \\
\hline Breast & & $-0.830 * * *$ & $-0.718^{* * *}$ & 0.035 & $-0.492 * * *$ & -0.238 & -0.070 & -0.173 & $0.657 * * *$ & 0.256 & 0.128 \\
\hline Thighs & & & 0.278 & -0.253 & 0.276 & 0.210 & 0.207 & 0.062 & $-0.370^{*}$ & -0.140 & -0.109 \\
\hline Wings & & & & 0.042 & $0.481 * *$ & 0.125 & -0.205 & 0.226 & $-0.784 * * *$ & -0.176 & $-0.317^{*}$ \\
\hline Ridge & & & & & $-0.468 * *$ & 0.093 & -0.191 & 0.263 & -0.080 & 0.153 & 0.021 \\
\hline Neck & & & & & & 0.224 & 0.192 & 0.001 & $-0.309^{*}$ & -0.266 & 0.102 \\
\hline Liver & & & & & & & $0.588 * * *$ & $0.374 * *$ & -0.198 & -0.091 & -0.061 \\
\hline Gizzard & & & & & & & & $0.332 *$ & 0.218 & -0.015 & -0.136 \\
\hline Heart & & & & & & & & & -0.257 & -0.185 & -0.020 \\
\hline $\begin{array}{l}\text { Abdomi- } \\
\text { nal fat }\end{array}$ & & & & & & & & & & $0.309^{*}$ & 0.193 \\
\hline
\end{tabular}

${ }^{*} p<0.05,{ }^{* *} p<0.01, * * * p<0.001$.

Table 5. Correlations between live weights, carcass weights, weights of carcass components, edible yields, abdominal fat weights, plasma triglyceride and glucose levels

\begin{tabular}{|c|c|c|c|c|c|c|c|c|c|c|c|c|}
\hline & Carcass & Breast & Thighs & Wings & Ridge & Neck & Liver & Gizzard & Heart & $\begin{array}{c}\text { Abdomi- } \\
\text { nal fat }\end{array}$ & $\begin{array}{l}\text { Trigly- } \\
\text { ceride } \\
(\mathrm{mg} / \mathrm{dL})\end{array}$ & $\begin{array}{l}\text { Glucose } \\
(\mathrm{mg} / \mathrm{dL})\end{array}$ \\
\hline $\begin{array}{c}\text { Live } \\
\text { weight }\end{array}$ & $0.964 * * *$ & $0.815^{* * *}$ & $0.945 * * *$ & $0.879 * * *$ & $0.846^{* * *}$ & $0.775 * * *$ & $0.525 * * *$ & 0.060 & $0.596 * * *$ & -.080 & 0.063 & 0.248 \\
\hline Carcass & & $0.892 * * *$ & $0.951 * * *$ & $0.866 * * *$ & $0.902 * * *$ & $0.762 * * *$ & $0.512 * * *$ & 0.066 & $0.597 * * *$ & 0.020 & 0.086 & $0.285^{*}$ \\
\hline Breast & & & $0.733 * * *$ & $0.606^{* * *}$ & $0.791 * * *$ & $0.560 * * *$ & $0.379 * *$ & 0.113 & $0.471 * *$ & $0.330 *$ & 0.183 & $0.301 *$ \\
\hline Thighs & & & & $0.868 * * *$ & $0.835^{* * *}$ & $0.758 * * *$ & $0.512 * * *$ & 0.048 & $0.571 * * *$ & -.0104 & 0.056 & 0.254 \\
\hline Wings & & & & & $0.799 * * *$ & $0.807 * * *$ & $0.529 * * *$ & -0.053 & $0.616 * * *$ & $-0.373 * *$ & -0.095 & 0.138 \\
\hline Ridge & & & & & & $0.578 * * *$ & $0.554 * * *$ & 0.048 & $0.616^{* * *}$ & -0.048 & 0.131 & 0.250 \\
\hline Neck & & & & & & & $0.480 * *$ & 0.085 & $0.505 * * *$ & -0.175 & -0.085 & $0.319^{*}$ \\
\hline Liver & & & & & & & & $0.390 * *$ & $0.523 * * *$ & -0.217 & -0.011 & 0.213 \\
\hline Gizzard & & & & & & & & & 0.239 & $0.313^{*}$ & 0.128 & 0.149 \\
\hline Heart & & & & & & & & & & -0.222 & -0.061 & 0.228 \\
\hline $\begin{array}{l}\text { Abdomi- } \\
\text { nal fat }\end{array}$ & & & & & & & & & & & $0.315^{*}$ & 0.220 \\
\hline
\end{tabular}

${ }^{*} p<0.05,{ }^{* *} p<0.01,{ }^{* * *} p<0.001$. 
males, partially agree with Cunningham et al. (1992) in which trimmed males at $6 \mathrm{wk}$ were significantly heavier than untrimmed males, whereas untrimmed females were heavier than trimmed females. Additionally, with respect to carcass weight, calculating heavier live weight in males than females was similar to Leeson and Summers (1980) in which males had heavier carcass weight in Large White turkeys. Veldkamp et al. (2000b) stated that percentage of carcass, breast and wing of male turkeys were $73.2,33.5$ and $11.7 \%$, respectively. In this study, percentage of carcass $(74.8 \%)$ and wings $(13.3 \%)$ of males were higher $(p<0.05)$ than the values reported by Veldkamp et al. (2000b), however, breast percentage (30.3\%) were lower. On the other hand, breast percentages of turkeys were reported as $32.6 \%$ in males and $34.2 \%$ in females (Blair et al., 1989). Similarly, in this study, breast percentage was higher $(p<0.05)$ for females. Stocking density (D) didn't affect significantly carcass percentage of turkeys but $\mathrm{D} \times \mathrm{S}$ interaction $(p<0.05)$ on carcass percentage. That means sex of the birds had difference response in different density. Halvorson et al. (1991) reported that the carcass percentage and carcass fat were affected by stocking density. Interaction of $\mathrm{B} \times \mathrm{D} \times \mathrm{S}$ in thighs indicating that thighs of birds were affected differently in different sex, density and trimmed or untrimmed condition.

Significant correlations were found between live weight with breast weight and thigh weight by (Havenstein et al., 1988). Similarly, in this study, it was determined that there are significant relations between live weight with breast weight and thigh weight $(p<0.001)$. The correlation between live weight and abdominal fat was insignificant. However, Barbour and Lilburn (1995) stated that a positive and linear relation between live weights with abdominal fat weight. On the other hand, low and positive correlations between live weight and abdominal fat in male (0.32) and female (0.25) turkeys were reported by Havenstein et al. (1988). There was a significant and positive relation between abdominal fat and plasma triglyceride level in turkeys in a study done by Bacon et al. (1989).

Beak trimming, sex separation, vaccination, and transport stress factors have changed blood glucose level in turkey poults (Donaldson et al., 1991). Nevertheless, Christensen et al. (2000) reported that there was a high correlation between hatching weight with blood glucose level and glucose concentration in blood increased by ageing. In this study, correlations between plasma glucose level with carcass weight, breast weight and neck weight were found as significant $(p<0.05)$. Plasma glucose level was affected by the $\mathrm{D} \times \mathrm{S}$ interaction.

\section{Conclusion}

Even tough, trimming did not have effect on live weight, carcass and its component significantly in general, the higher live weight and carcass weight observed in trimmed groups. As expected, male of birds is heavier than female, and carcass percentage would be adverse. However, in this study, carcass percentage of male was higher in trimmed, in $0.25 \mathrm{~m}^{2} / \mathrm{bird}$. That means trimming could be used with stocking density. It would be expected that the higher triglyceride, the higher abdominal fat around liver. The highest triglyceride level was observed in female of trimmed group, in $0.5 \mathrm{~m}^{2} /$ bird. More study needs to be done to figure out clear effect of trimming on triglyceride.

\section{References}

1. Bacon, W. L., Nestor, K. E., and Naber, E. C. (1989) Prediction of carcass composition of turkeys by blood lipids. Poultry Sci. 68, 1282-1288

2. Barbour, G. W. and Lilburn, M. S. (1995) Characterization of carcass development from 14 to 145 days of age in turkey hens from two strains. Poultry Sci. 74, 1650-1658.

3. Blair, M. E., Potter, L. M., and Hulet, R. M. (1989) Effects of dietary protein and added fat on turkeys varying in strain, sex, and age. 2. Carcass characteristics. Poultry Sci. 68, 287296.

4. Brake, J., Havenstein, G. B., Ferket, P. R., Rives, D. V., and Giesbrecht, F. G. (1995) Relationship of sex, strain, and body weight to carcass yield and offal production in turkeys. Poultry Sci. 74,161-168.

5. Christensen, V. L., Grimes, J. L., Donaldson, W. E., and Lerner, S. (2000) Correlation of body weight with hatchling blood glucose concentration and its relationship to embryonic survival. Poultry Sci. 79, 1817-1822.

6. Cunningham, D. L., Buhr, R. J., and Mamputu, M. (1992) Beak trimming and sex effects on behavior and performance traits of Large White Turkeys. Poultry Sci. 71, 1606-1614.

7. Donaldson, W. E., Christensen, V. L., and Krueger, K. K. (1991) Effects of stressors on blood glucose and hepatic glycogen concentrations in turkey poults. Comp. Biochem. Physiol. A. 100, 945.

8. Ensminger, M. E. (1992) Poultry Sci. Third Edition, Interstate Publishers, INC., Danville, Illinois, pp. 255-280, 329354.

9. Halvorson, J. C., Waibel, P. E., Oju, E. M., Noll, S. L., and El Halawani, M. E. (1991) Effect of diet and population density on male turkeys under various environmental conditions. 2. Body composition and meat yield. Poultry Sci. 70, 935-940.

10. Havenstein, G. B., Toelle, V. D., Nestor, K. E., and Bacon, W. L. (1988) Estimates of genetic parameters in turkeys. 2. Body weight and carcass characteristics. Poultry Sci. 67, 13881399. 
11. Leenstra, F. R. (1986) Effect of age, sex, genotype and environment on fat deposition in broiler chickens. World's Poultry Sci. J. 42, 12-25.

12. Leeson, S. and Summers, J. D. (1980) Production and carcass characteristics of the Large White turkey. Poultry Sci. 59, 1237-1245.

13. Lilburn, M. S. and Nestor, K. E. (1991) Body weight and carcass development in different lines of turkeys. Poultry Sci. 70, 2223-2231.

14. North, M. O. and Bell, D. D. (1990) Commercial Chicken Production Manual. 4th ed. Chapman Hall, New York.

15. Parkhurts, C. R. and Mountney, G. J. (1988) Poultry, meat and egg production. D. van Nostrand Company Inc., New York.
16. Sell, J. L., Hasiak, R. J., and Owings, W. J. (1985) Independent effects of dietary metabolizable energy and protein concentrations on performance and carcass characteristics of tom turkeys. Poultry Sci. 64, 1527-1535.

17. Veldkamp, T., Ferket, P. R., Kwakkel, R. P., Nixey, C., and Noordhuizen, J. P. (2000a) Interaction between ambient temperature and supplementation of synthetic amino acids on performance and carcass parameters in commercial male turkeys. Poultry Sci. 79, 1472.

18. Veldkamp, T., Kwakkel, R. P., Ferket, P. R., Simons, P. C., Noordhuizen, J. P., and Pijpers, A. (2000b). Effects of ambient temperature, arginine-to-lysine ratio, and electrolyte balance on performance, carcass, and blood parameters in commercial male turkeys. Poultry Sci. 79, 1608-1616. 